\title{
ESTUDIOS DE CUERPOS Y CORPORALIDADES EN CHIAPAS: UNA RESPUESTA A LAS LIMITACIONES DE LA CATEGORÍA GÉNERO EN EL ESTUDIO DE LAS REALIDADES INTERSECCIONADAS DE LAS MUJERES
}

\section{Study of Bodies and Corporalities in Chiapas: A Response to the Limits of THE Gender Category in the Study of Women's Intersectional Reality}

\author{
Lorena Álvarez-Ossa*
}

DOI: http://dx.doi.org/10.29043/liminar.vl9il.810

Reseña de Cuerpo y política. Feminismos, género e interseccionalidad, editado por Inés Castro Apreza. Tuxtla Gutiérrez: Universidad de Ciencias y Artes de Chiapas-Centro de Estudios Superiores de México y Centroamérica, 2019. En https://repositorio.cesmeca.mx/handle/11595/969 (consultado el 10 de agosto de 2020).

$\mathrm{E}$ 1 libro Cuerpo y política. Feminismos, género e interseccionalidad, editado por Inés Castro Apreza (2019), es un trabajo original porque busca avanzar teórica y conceptualmente en los recientes estudios sobre cuerpo y corporalidades en Chiapas, en parte como respuesta académica a las limitaciones de la categoría género para la comprensión de las realidades interseccionadas de las mujeres, particularmente indígenas, campesinas y mestizas urbanas empobrecidas, cuya existencia está marcada por distintos sistemas de opresión, clasistas, racistas, sexistas, heteronormativos. También para construir memoria del trabajo realizado a propósito, desde coordenadas feministas, en el sureste de México. Por lo tanto, este libro, tal como lo presenta la editora, es una apuesta teórica, metodológica, epistémica y política que posiciona la escritura de mujeres académicas y militantes, a partir de experiencias propias, valorando una perspectiva epistemológica situada y encarnada, desde una mirada multidisciplinar que incluye la antropología, la sociología, la política y la psicología.

* Lorena Álvarez Ossa. Magister en Desarrollo Universidad Pontificia Bolivariana (UPB), Colombia. Doctorante en Ciencias Sociales y Humanísticas en el Centro de Estudios Superiores de México y Centroamérica de la Universidad de Ciencias y Artes de Chiapas (CESMECA-UNICACH). Temas de especialización: feminismos, mujeres negras racializadas,
Empecemos por lo segundo. En este aspecto, es importante dar cuenta de cómo en este libro existe un importante esfuerzo por recoger los avances en términos experienciales, empíricos y teóricos, que se han formulado después del precedente fundacional - por lo que implicó y sigue implicando- que representa el Coloquio Cuerpos y Diversidades, llevado a cabo en el año 2014 entre la Universidad de Ciencias y Artes de Chiapas (UNICACH) y la Universidad Intercultural de Chiapas (UNICH), cuyas memorias constituyen una fuente obligatoria de consulta sobre el tema (Castro y Morales, 2016). En efecto, como lo explica Castro, los estudios sobre cuerpos y corporalidades aún están desplegando su potencial en Chiapas, y su futuro depende de la consolidación de cuerpos docentes especializados en el tema, del direccionamiento y de la motivación de jóvenes tesistas hacia esta área de conocimiento, así como de la realización de cursos y seminarios especializados, apuesta en la que la memoria, las genealogías de las trayectorias y el trabajo académico y militante son fundamentales, en tanto constituyen el horizonte

cuerpos y corporalidades como estrategia de resistencia a la lógica de la guerra. Correo electrónico: lorena.alvarez071@gmail.com. ORCID: http:// orcid.org/0000-0001-7829-7893

Recibida: 18 de agosto de 2020.

Aprobada: 15 de septiembre de 2020. 
de sentido teórico, metodológico y político en el cual se inscribe esta compilación.

En el sentido anterior, de esa actividad académica en particular, Castro subraya lo cardinal del recorrido hecho por las nociones de cuerpo y corporalidad, destacando el trabajo de Tania Cruz (2016), quien significa el cuerpo como un espacio de desigualdades sociales que se moldean con la apariencia y el comportamiento, a través del cual circulan normas, discursos e imágenes que están en tensión entre la imposición y la existencia. Cruz no desconoce la importancia de la categoría género; empero, evidencia su insuficiencia, por lo que acoge una perspectiva de interseccionalidad que le ofrece la posibilidad de conectar las desigualdades, para el estudio de la corporeidad y las prácticas de embellecimiento de las jóvenes chipanecas, que pasan necesariamente por el cuerpo como un lugar de historias personales, de construcción de la identidad, clasificación y distinción. A esto se suma una serie de referencias a otros estudios novedosos producidos recientemente en la región: el de Liliana Bellato (2016) sobre los cuerpos de mujeres y hombres mayores y los discursos de saber/poder sobre la menopausia y la erección, como acentos de una sexualidad manipulada que se sostiene en un binarismo de género y está imbricada en un sistema heteropatriarcal, heterosexual y capitalista; el de Montserrat Bosch (2016), quien construye una noción epistemológica situada al tomar el propio cuerpo como lugar de análisis, asunto reflejado en los estudios de quien observa su propio cuerpo femenino afectado por el cáncer de mama y metamorfoseado a partir del poder biomédico y las nociones de saber/poder que constituyen la subjetividad femenina, a partir de la heterosexualidad normalizada y ordenada a las mujeres; y el de Ixchel Muñoz (2016), quien conceptualiza el cuerpo desde la experiencia de aborto, tema fundamental hoy en día en las agendas feministas, al que se concibe como una construcción social, que está marcada por su posicionamiento feminista.

Asimismo, el libro Cuerpo y política. Feminismos, género e interseccionalidad responde a la necesidad, de cara a la difusión y consolidación de los estudios feministas y de género, de abordar las realidades de los sujetos -en este caso, las mujeres-, complejizando la utilización de la perspectiva teórica de interseccionalidad, que si bien no aparece explícitamente en algunos apartados de este libro, se hace evidente en su uso, cuando se tratan cuestiones no solo referidas a la interesección etnia/raza, género o clase social, sino también cuando se pregunta por la sexualidad, la edad, la religiosidad, la pertenencia urbana/rural, entre otros temas. A este respecto, es relevante nombrar que, desde la introducción de la categoría de interseccionalidad con Kimberly Crenshaw a finales de los años 1980, se ha enriquecido la teoría feminista, al abrirse una polifonía de voces de mujeres que no se reflejan en la cuadrícula de mujer singular o mujeres en plural construida por el feminismo blanco occidental, que presenta a las mujeres solo como víctimas y en tutela de los hombres. Las aportaciones más significativas en este sentido, como sabemos y siempre vale la pena recordar, son de las feministas negras -Colectiva Río Combahee (1988), Angela Davis, Audre Lorde, Patricia Hill Collins (2000), bell hooks y Kimberlé Crenshaw, entre otras - descoloniales y antirracista - Chandra Talpade Mohanty, María Lugones (2005), Ochy Curiel, Yuderkis Espinosa, Soeli Carneiro y Léila González, entre otras-, quienes en las últimas décadas han problematizado y alimentado estos debates, ampliando la comprensión y derivando otras conceptualizaciones.

Entonces, en la dinámica de ampliar la comprensión de la realidad de las mujeres, en Cuerpo y política. Feminismos, género e interseccionalidad subyacen preguntas por el cuerpo y las corporalidades de las mujeres y de otras personas que se presentan como subalternas en una dimensión interseccional: ¿a quiénes pertenecen los cuerpos de las mujeres?, ¿al servicio de qué intereses se socializan y ponen estos cuerpos?, ¿son autónomos en su capacidad de agencia?, ¿qué margen de agencia tienen para crear y recrear sus propias realidades?, ¿hay posibilidad de escapar a los corsés normativos marcados por la religión, la familia, la educación, la clase social, la posición de bienes materiales e inmateriales, entre otros?

A propósito, y como he anunciado, los análisis que se presentan se extraen de las vivencias de mujeres concretas desde sus propias cotidianidades y sus prácticas organizativas. Es el caso del estudio y la observación del 
ministerio femenino de la pastora Sol Corzo de la Iglesia cristiana, en el que Karla Lizbeth Somosa (pp. 47-86) se esfuerza por visibilizar los mecanismos religiosos que disciplinan el cuerpo de las mujeres de la congregación, a los que no escapa la misma pastora. También la experiencia de un albergue para víctimas de violencia sexual y doméstica, La Morada contra la violencia sexual y doméstica, que presentan Blanca Olivia Velázquez y Georgina Sánchez (pp. 123-146), que recoge, entre otros aspectos, los obstáculos que presenta el desarrollo de un programa de atención de violencia contra las mujeres por la burocratización de las instancias de género del Estado y el despojo de su carácter político feminista, lo que limita su actuar a acciones asistencialistas que reproducen las desigualdades sociales y de género. El examen de las acciones legales interpuestas por trabajadoras sexuales para exigir que se les reconozca como trabajadoras en México, es el tema que presenta Cruz Yolanda Martínez (pp. 147-174), quien establece los dilemas de la intervención estatal en la actividad prostibularia, señalando que una inadecuada regulación de esta actividad hace que las personas que la ejercen queden expuestas a mayor vulneración, discriminación y riesgo de control y abuso por parte de proxenetas, así como a las extorsiones de autoridades y policías corruptos, entre otros. También destaca el caso de una mujer indígena víctima de violencia sexual, a través del cual Mariel Cameras Myers (pp. 175-196) identifica la ocupación, cooptación e instrumentalización del cuerpo de las mujeres por los hombres a través de las relaciones de poder de género presentes en las prácticas de justicia indígena.

En esta misma idea, Lilliana Bellato Gil (pp. 105122) muestra el cuerpo como espacio de heterotopía en términos foucaultianos, como lugar de exclusión/ inclusión, de transgresión y de reafirmación de la norma, abordando un tema tabú, el erotismo de personas mayores, en una realidad marcada por el desprecio a la vejez y el deseo de la eterna juventud; identifica, a su vez, las vivencias de hombres y mujeres mayores, a quienes se les expropia de su sexualidad, lo que se justifica en discursos de "la impertinencia estética" y de faltas a la moral. Desde otro ángulo, Astrid Maribel Pinto (pp.
87-104) presenta la experiencia de un grupo espiritual caracterizado por una práctica ecuménica, abierta a todos los credos y creencia, que no pretende constituirse como institución religiosa y a su vez deslegitima y rechaza todas las instituciones de esta naturaleza.

Es importante, al mismo tiempo, exaltar y reconocer el trabajo etnográfico de las autoras, que constituyen un ejemplo de cómo hacer investigación empírica feminista subvirtiendo la formar en la que se investiga, ello considerando elementos como: la relevancia de la identidad del sujeto en la producción de conocimiento - en este caso particular, las mujeres-, el cuestionamiento del vigor de las normas metodológicas que aparecen como objetivas e inapartables de los procesos de investigación, y el acento sobre la relación entre ciencia y policía, proponiendo una política emancipadora que fortalece y amplía la objetividad de la ciencia, asuntos tratados por Sandra Harding (1996) y otras en los estudios de epistemología, método y metodología feminista. En este punto, es interesante citar a Donna Haraway (1991) y sus debates sobre la naturaleza y el poder de la producción del conocimiento, desde los que la autora cuestiona la visión que de lo femenino se observa en la investigación tradicional, así como los universales absolutos y las matrices de unidad, universalidad y verdad total.

En conclusión, en este libro se abren novedosas preguntas sobre las realidades y cotidianidades de los sujetos desde una perspectiva epistemológica feminista situada de las investigadoras. Los ensayos responden a aspectos políticos, sociales, culturales y económicos sobre cómo pueden comprenderse las relaciones de poder que viven las mujeres, particularmente indígenas, campesinas y mestizas urbanas empobrecidas, afectadas por la desigualdad y la discriminación, y como desde allí, desde ese lugar de subalternidad, aparece y se dibuja su capacidad de agencia.

\section{Referencias}

Bellato Gil, Liliana (2016). “Los cuerpos hablan. Más allá de los mitos de la menopausia y la erección entre mujeres y hombres mayores". En Inés Castro Apreza y Susie Morales Moreno (coords.). Cuerpos y diversida- 
des. Miradas desde el sur. Tuxtla Gutiérrez: Universidad de Ciencias y Artes de Chiapas-Centro de Estudios Superiores de México y Centroamérica, Universidad Intercultural de Chiapas, pp. 171-198. En https://repositorio.cesmeca.mx/handle/11595/851 (consultado el 10 de agosto de 2020).

Bosch Heras, Montserrat (2016). "Las nuevas amazonas. Mujeres tras la mastectomía”. En Inés Castro Apreza y Susie Morales Moreno (coords.). Cuerpos y diversidades. Miradas desde el sur. Tuxtla Gutiérrez: Universidad de Ciencias y Artes de Chiapas-Centro de Estudios Superiores de México y Centroamérica, Universidad Intercultural de Chiapas, pp. 161-170. En https://repositorio.cesmeca.mx/handle/11595/851 (consultado el 10 de agosto de 2020).

Castro Apreza, Inés (2019). Cuerpo y política. Feminismos, género e interseccionalidad. Tuxtla Gutiérrez: Universidad de Ciencias y Artes de Chiapas-Centro de Estudios Superiores de México y Centroamérica. En https:// repositorio.cesmeca.mx/handle/11595/969 (consultado el 10 de agosto de 2020).

Castro Apreza, Inés y Susie Morales Moreno (coords.) (2016). Cuerpos y diversidades. Miradas desde el sur. Tuxtla Gutiérrez: Universidad de Ciencias y Artes de Chiapas-Centro de Estudios Superiores de México y Centroamérica, Universidad Intercultural de Chiapas. En https://repositorio.cesmeca.mx/handle/11595/851 (consultado el 10 de agosto de 2020).

Colectiva Río Combahee (1988). "Una declaración feminista negra”. En Cherrie Moraga y Ana Castillo (eds.), Esta puente, mi espalda. Voces de mujeres tercermun- distas en los Estados Unidos. San Francisco: ism press, pp. 172-186.

Collins, Patricia Hill (2000). Pensamiento feminista negro: el conocimiento, la conciencia y la política de empoderamiento. Nueva York: Routledge.

Cruz Salazar, Tania (2016). "Reflexiones en torno a los estudios del cuerpo”. En Inés Castro Apreza y Susie Morales Moreno (coords.). Cuerpos y diversidades. Miradas desde el sur. Tuxtla Gutiérrez: Universidad de Ciencias y Artes de Chiapas-Centro de Estudios Superiores de México y Centroamérica, Universidad Intercultural de Chiapas, pp. 34-50. En https://repositorio.cesmeca.mx/handle/11595/851 (consultado el 10 de agosto de 2020).

Harding, Sandra (1996). Cienciay feminismo. Madrid: Morata. Haraway, Donna (1991). Ciencia, cyborgs y mujeres. La reinvención de la naturaleza. Madrid: Cátedra, Universidad de Valencia, Instituto de la Mujer.

Lugones, María (2005). "Multiculturalismo radical y feminismos de mujeres de color". En Revista Internacional de Filosofía Política, 25, 61-76.

Muñoz Soto, Fabiola Ixchel (2016). "Del cuerpo normado al cuerpo transgresor. Experiencias de aborto en San Cristóbal de Las Casas". En Inés Castro Apreza y Susie Morales Moreno (coords.). Cuerpos y diversidades. Miradas desde el sur. Tuxtla Gutiérrez: Universidad de Ciencias y Artes de Chiapas-Centro de Estudios Superiores de México y Centroamérica, Universidad Intercultural de Chiapas, pp. 307-321. En https://repositorio.cesmeca.mx/handle/11595/851 (consultado el 10 de agosto de 2020). 